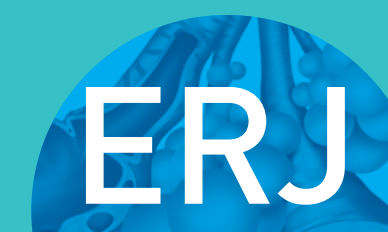

open research
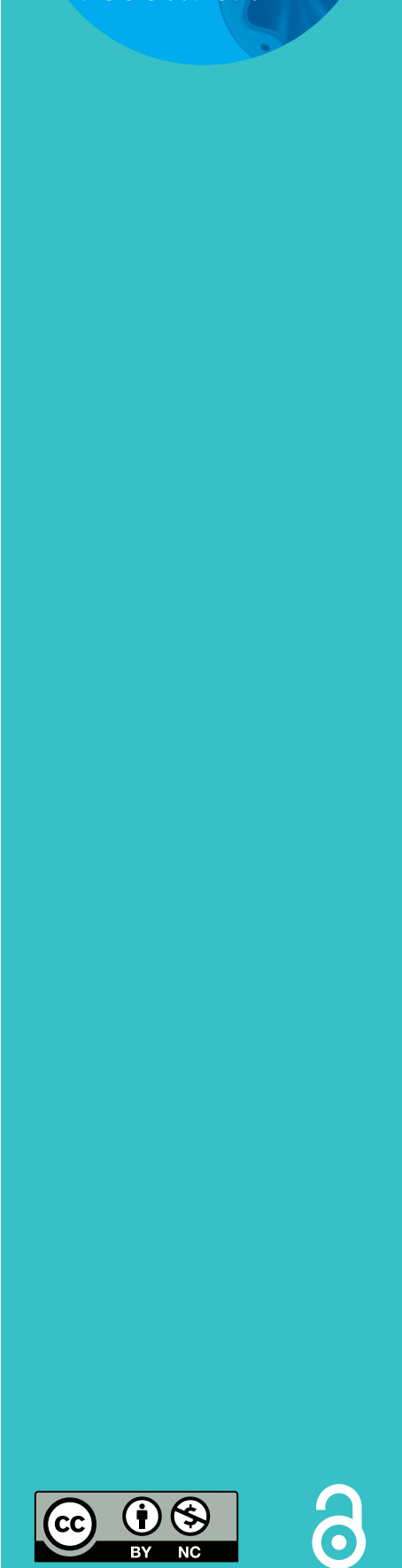

\section{High-altitude alpine therapy and lung function in asthma: systematic review and meta-analysis}

\author{
Denis Vinnikov ${ }^{1}$, Abdullah Khafagy ${ }^{2,3}$, Paul D. Blanc ${ }^{2}$, Nurlan Brimkulov ${ }^{1}$ and \\ Craig Steinmaus ${ }^{4}$
}

Affiliations: ${ }^{1}$ Dept of Internal Medicine, Occupational Diseases and Hematology, Kyrgyz State Medical Academy, Bishkek, Kyrgyz Republic. ${ }^{2}$ Division of Occupational and Environmental Medicine, University of California San Francisco, San Francisco, CA, USA. ${ }^{3}$ Community Medicine and Pilgrims Healthcare Dept, Umm Al-Qura University College of Medicine, Mecca, Saudi Arabia. ${ }^{4}$ School of Public Health, University of California Berkeley, Berkeley, CA, USA.

Correspondence: Denis Vinnikov, Dept of Internal Medicine, Occupational Diseases and Hematology, Kyrgyz State Medical Academy, Akhunbaev Street 92, Bishkek 720020, Kyrgyzstan. E-mail: denisvinnikovamail.ru

ABSTRACT We used meta-analysis to measure the effect of high-altitude climate therapy (HACT) on lung function outcomes in asthma, and systematically searched PubMed, Embase and www.elibrary.ru for publications appearing from 1970 to mid-2015. We included studies carried out with children or adults with an exposure of up to 12 weeks at an altitude of $\geqslant 1500 \mathrm{~m}$ above sea level. Changes in forced expiratory volume in $1 \mathrm{~s}$ (FEV1), FEV1/vital capacity ratio or peak expiratory flow rate as the HACT intervention outcomes were analysed. We included data for 907 participants (age range 4-58 years) from 21 studies, altogether including 28 substrata based on asthma type or severity. Only three of 21 included studies had high quality, whereas $93 \%$ of substudies reported lung function improvement with an overall pooled standardised mean difference (SMD) of 0.53 (95\% CI 0.43-0.62). The measured effect of HACT was greater in adults (SMD 0.75, 95\% CI 0.63-0.88, $\mathrm{n}=14$ ) than in children (SMD 0.24, 95\% CI 0.09-0.38, $\mathrm{n}=14$ ). Studies at altitude $>2000 \mathrm{~m}$ above sea level yielded the same effect as those at lower altitude. Based on a cut-point of a 0.50 change in SMD to define a meaningful clinical difference, HACT appears to have efficacy as an intervention. This extent of benefit appears to be limited to adults with asthma.

@ERSpublications

High-altitude alpine therapy may be an effective intervention to improve lung function in patients with asthma http://ow.ly/u3i23008vU5

\section{Conflict of interest: None declared.}

Copyright CERS 2016. This article is open access and distributed under the terms of the Creative Commons Attribution Non-Commercial Licence 4.0. 


\section{Introduction}

Environmental modification remains a key component of asthma management, ranked together with appropriate maintenance pharmacologic therapy in stepwise treatment protocols [1]. Environmental interventions often begin with household allergen reduction (e.g. dust mite controls) or irritant removal (e.g. avoidance of second-hand cigarette smoke). More advanced environmental modification strategies also have an established role in the care of children and adults with asthma. Examples include speleotherapy, hypobaria and, most notably, high-altitude climate therapy (HACT, also known as "alpine" therapy). Historically, this latter treatment is the most widely used single modality of such environmental modulation for asthma [2]. Due to inconvenience, expense and questionable efficacy, none of these environmental interventions is currently accepted widely, including HACT. Nevertheless, there are biological mechanisms through which a beneficial effect of HACT is plausible, including reduced antigen exposure [3, 4], immune modulation $[5,6]$ and lowered air viscosity possibly ameliorating airway resistance.

Multiple investigators have studied the effects of HACT at various treatment sites around the world, characteristically studies that were not blinded or properly randomised and that provided single-arm, post-treatment to pre-treatment comparisons. Elements of the HACT literature are poorly accessible for critical review, however; in particular due to non-English language publication. These accumulated data present a rich opportunity for a systematic assessment of the presence or absence of treatment efficacy for HACT. We wished to address the existing knowledge gap in the treatment effect of HACT for asthma by subjecting data from these studies to a formal meta-analysis, including publications that had not appeared in the English language literature.

\section{Methods}

Data sources and searches

Two researchers (D.V. and A.K.) independently performed the initial literature search and abstract screening. For differences in initial assessment necessitating adjudication, we strove for consensus among all four co-authors. The search was completed in August 2015 and utilised three databases: PubMed, Embase and a major Russian resource, www.elibrary.ru. The search was limited to publications appearing from 1970 onwards. We used the following search term strategies: "asthma” AND "altitude" in Embase; asthma(Title/ Abstract) AND altitude(MeSH Terms "altitude" including subheadings: adverse effects, epidemiology, history, instrumentation, pharmacology, physiology, statistics and numerical data, therapeutic use, and therapy" OR "altitude sickness" OR "pulmonary edema of mountaineers") in PubMed; and "asthma" AND "high-altitude" OR "high-altitude climate therapy" OR "alpine therapy" (in Russian) for www.elibrary.ru. The results of the search algorithm using the PRISMA protocol, and consistent with its checklist and steps of study identification and inclusion, are shown in figure 1 [7].

\section{Study selection, data extraction and assessment of risk of bias}

PubMed, Embase and www.elibrary.ru were searched, returning 342 cited publications that appeared from 1970 onwards. We considered eligible studies that represented original research on human asthma treatment involving exposure to conditions of altitude $\geqslant 1500 \mathrm{~m}$ above sea level (MASL). There was no age restriction: studies both of adult and childhood asthma were considered eligible. We also searched the references cited by papers in a systematic review [8]. Of 342 citations identified initially, 307 (90\%) did not meet the eligibility criteria at the initial screening stage. The remaining 35 studies underwent further review. Critical review of complete texts of the 35 eligible studies identified two publications $[9,10]$ that were excluded because they were based on data ultimately subsumed in two other publications $[11,12]$. We also found that a study set of one publication [13] was included within a larger cohort [14], so only the latter was included. This produced 32 eligible papers for this review. At this stage of review, we further excluded initially eligible studies that did not include at least one of the following measures derived from pulmonary function testing: forced expiratory flow in $1 \mathrm{~s}$ (FEV1), the ratio of FEV1 to forced vital capacity (FVC) or vital capacity (VC), or peak expiratory flow rate (PEFR). There were nine studies excluded due to a lack of such pulmonary function data. We additionally excluded two other studies that only examined longer-term effects of HACT (>12 weeks duration at altitude). After all exclusions, 21 publications remained in the final meta-analysis.

Quality assessment using available scales was hard to accomplish because of significant heterogeneity arising from absence of uniform criteria for performing such studies across different locations and time. Therefore, we set our own criteria to assess quality of studies, and minimum quality parameters for study inclusion were pulmonary function measurements before and during altitude, no evidence of dropout rates $>60 \%$, no other major reported change in therapy, and altitude of at least 1500 MASL.

\section{Data synthesis and analysis}

The end-point for analysis was one of the measures of pulmonary function required for eligibility. For studies that reported more than one pulmonary function end-point, we preferentially selected the FEV $1 \%$ 


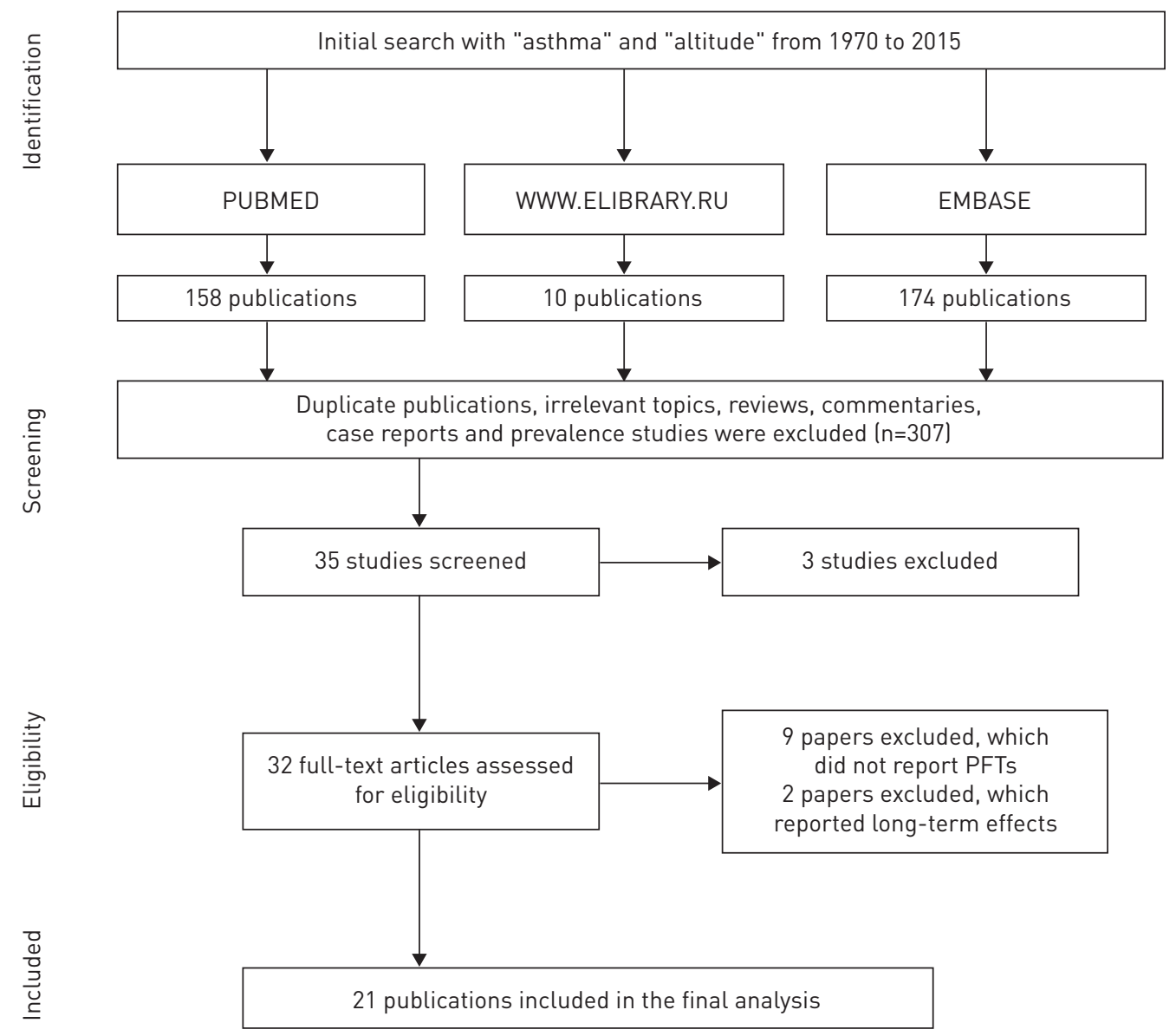

FIGURE 1 Search algorithm. PFT: pulmonary function test.

pred for analysis. If the raw FEV1 only was available rather than as a percent predicted, we extracted that outcome. If these were not provided, we selected the PEFR (actual or as a percent predicted). The FEV1/ FVC (or VC) ratio was used only if neither of the other two measures was available. The pool of identified studies included both randomised controlled studies and single-arm studies without a control group. For consistency, we extracted the pulmonary function end-point of interest before and after altitude exposure and did not utilise data from control study arms (i.e. study of persons without asthma at altitude). We calculated the mean difference in end-point between the baseline pre-treatment value and after the high-altitude intervention. The pre-treatment value could represent measurement at low altitude or immediately upon arrival at high altitude. Several studies stratified patients based on asthma severity (mild, moderate or severe) or aetiology (atopic and nonatopic asthma), reporting grouped pulmonary function data within such strata. In those cases, because they represented independent samples (an assumption of the meta-analysis model), we treated each stratum as a separate substudy. Taking such strata into account, among the 21 studies included we ultimately analysed 28 end-points.

The effect size was calculated as the mean change in the selected pulmonary function end-point subtracting the baseline value from the post-treatment value. Thus, a positive mean value represented a treatment-associated improvement in lung function. In addition to studies reporting effect sizes by varying scales (i.e. using differing lung function indices), we calculated the standardised mean differences (SMDs). A few studies reported the standard error rather than the standard deviation. For those studies, we derived the standard deviation from the reported standard error using the formula: $S D=S E \times n^{1 / 2}$. For a single study reporting the median value of pulmonary function end-point together with its $95 \%$ confidence interval, we calculated the standard deviation assuming the mean to be the mid-point of the $95 \%$ confidence interval, using the formula: $\mathrm{SD}=\mathrm{n}^{1 / 2} \times((95 \%$ upper bound-mean $) / 1.96)$. We used Stata 14.1 software to run a meta-analysis function to calculate SMDs. We recalculated SMDs in stratified analyses based on age, altitude and duration of study. In a sensitivity analysis, we calculated the nonstandardised outcome for the pool of studies reporting mean differences in FEV1 \% pred. Further sensitivity analyses recalculated the pooled SMD after selected exclusions on studies to assess their impact on the overall results. Publication 
bias was assessed using Egger's and Begg's tests $[15,16]$. The degree of heterogeneity due to between-study variability was estimated using the Chi-squared test with the relevant p-value. For interpretation purposes, heterogeneity was also supplemented with the $I^{2}$ metric [17]. We also report the tau value $\left(\tau^{2}\right)$ in absolute units as an estimate of heterogeneity among the studies.

\section{Results}

Table 1 summarises the 21 studies included in the analysis. These subsumed, in total, 28 strata treated as independent substudies for the purposes of the meta-analysis. All but four studies reported the FEV1 \% pred, allowing us to analyse this in the majority of cases (17 of 21 included studies; 21 of 28 substudy strata). Data for raw FEV1 and for PEFR were available from one study each, and the FEV1/VC ratio from two others (altogether end-points other than FEV1 \% pred from seven substudy strata). In total, data for 907 participants were included, of whom 568 (63\%) were adults. 13 studies were conducted in Western Europe (Italy and Switzerland), four in Eastern Europe, three in Nepal and one in South America. The publication dates for the studies ranged from 1973 to 2013; 12 (57\%) were published before 2000. In all but one study [11], pre-altitude asthma pharmacotherapy was continued when exposure to altitude was initiated. Such baseline therapy was heterogeneous, varying from patient to patient and over time with changing standards of care. Short-acting $\beta$-agonists were typical across studies as rescue medication.

As shown in figure 2, the SMD post-treatment ranged from a fall in lung function of -1.79 (95\% CI $-3.31--0.27)$ to an increase of 2.96 (95\% CI 2.36-3.56) standardised units. Heterogeneity across studies was high $\left(\tau^{2}=1.58, I^{2}=85 \%, \mathrm{p}<0.001\right)$. Despite this statistical heterogeneity, the studies were mostly consistent in their direction of effect. In total, 26 of 28 strata (93\%) observed a positive response (SMD $>0$ ), while a lung function reduction was observed in only two strata. Of note, in both of these, which accounted for only $3.2 \%$ of the overall study weight, the exposure was to altitudes $>4000$ MASL [31, 33]. Of the 26 positive studies/substudies, 13 yielded an SMD $>0$ but $<0.50$, while 13 were at or above that value, which is an accepted threshold for a meaningful clinical difference (MCD) [37, 38]. Of these, eight yielded an SMD of $\geqslant 0.50$ or more but $\leqslant 1.0$, while for six, the SMD was $>1.0$. The studies/substudies yielding a positive treatment effect size of $\geqslant 0.5$ SMD subsumed 403 of the 907 (44\%) subjects in the total study pool. There was a trend to more attenuated effects among the publications with a more recent year of publication (figure 2).

In order to investigate further potential differences in HACT efficacy by intervention and by study population, we carried out stratified analyses by study site altitude, age group (childhood versus adult) and treatment duration. These stratified analyses are shown in table 2. Six studies/substudies carried out at an altitude >2000 MASL reported a lung function improvement (SMD 0.53, 95\% CI 0.32-0.74) that was similar to that seen in the 22 studies/substudies carried out at an altitude of $\leqslant 2000$ MASL (SMD 0.53, 95\% CI $0.42-0.64)$ ). Stratification by age (children versus adults) yielded a positive effect in both groups, although the estimated SMD was greater in adults (SMD 0.75, 95\% CI 0.63-0.88) compared with that among children (SMD 0.24, 95\% CI 0.09-0.38). Treatment for $>4$ weeks was associated with a marginally greater effect size than more brief treatment (SMD 0.63, 95\% CI $0.50-0.77$ versus SMD $0.42,95 \%$ CI $0.28-0.55$, respectively). In the single study in which baseline medicines were discontinued, the observed SMD was small and not statistically significant (SMD 0.10, 95\% CI -0.73-0.94).

We performed a sensitivity analysis excluding studies reporting outcome measures other than FEV1 \% pred ( $\mathrm{n}=7$ studies/substudies excluded). After this exclusion, the overall SMD was somewhat lower (SMD 0.34, 95\% CI 0.24-0.45). As these studies reported effect using the same scale, we also calculated the mean difference in this stratum without standardisation. At baseline, FEV1 \% pred ranged from $64.5 \%$ to $105.8 \%$ (median 90.2\%). The overall FEV1 \% pred increase was 5.79\% (95\% CI 4.36-7.23) with moderate heterogeneity $\left(\tau^{2}=0.29, I^{2}=58 \%, p<0.001\right)$. A forest plot of this analysis is presented in figure 3 . In a $170 \mathrm{~cm} \mathrm{30}$-year-old adult male, this would equate to $229 \mathrm{~mL}$ (assuming FEV 1 100\% predicted to be $3.95 \mathrm{~L}$ ) [36]. We also carried out another sensitivity analysis by re-estimating the effect of HACT excluding each individual study one by one. This re-analysis showed that the study by DubiLEY et al. [18] made the biggest contribution to the overall SMD: when excluded (all three substudies from that publication combined), the overall SMD fell to 0.35 , but was still statistically significant (95\% CI 0.25-0.46) (table 3). In the opposite direction, the study of VeRKLEI et al. [14] brought the pooled SMD down to the greatest degree: when excluded, the overall SMD increased to 0.60 (95\% CI 0.49-0.70). When excluded, the only study that had reported lung function as a median rather than as a mean value [30] did not substantively change the observed overall SMD $(0.55,95 \%$ CI $0.45-0.65)$. We also wished to calculate SMD for studies performed at altitudes of $>2500$ MASL, which is an accepted threshold for high altitude. Six remaining substudies from altitudes of 3200-6965 MASL yielded SMD 0.53 (95\% CI 0.32-0.74). Finally, when studies that used control group designs were excluded in order to determine if these had impacted the findings even though the control arm data had not been included [26, 31, 33], the pooled SMD slightly increased 
TABLE 1 Characteristics of the 22 studies including 29 substrata included in the meta-analysis

\begin{tabular}{|c|c|c|c|c|c|c|c|c|c|c|c|c|}
\hline Study & Strata & $\begin{array}{l}\text { Study and year of } \\
\text { publication }\end{array}$ & $\begin{array}{l}\text { Study location } \\
\text { (language of } \\
\text { publication) }\end{array}$ & $\begin{array}{l}\text { Altitude } \\
\text { MASL }\end{array}$ & $\begin{array}{l}\text { Patient } \\
\text { group } \\
\text { adults } \\
\text { versus } \\
\text { children }\end{array}$ & $\begin{array}{l}\text { Subjects } \\
\text { (subgroup) }\end{array}$ & $\begin{array}{l}\text { Outcome } \\
\text { measure } \\
\text { included in } \\
\text { analysis }\end{array}$ & $\begin{array}{l}\text { Observed } \\
\text { change }\end{array}$ & $\begin{array}{c}\text { Mean } \\
\text { difference }\end{array}$ & SMD & $\begin{array}{l}\text { Treatment } \\
\text { duration and } \\
\text { additional } \\
\text { comments }\end{array}$ & $\begin{array}{l}\text { Quality of } \\
\text { evidence }\end{array}$ \\
\hline \multirow[t]{3}{*}{1} & 1 & $\begin{array}{l}\text { DubiLEY and } \\
\text { SHOGENTSUKovA, } \\
1973 \text { (1) [18] }\end{array}$ & $\begin{array}{c}\text { USSR } \\
\text { (Russian) }\end{array}$ & 2000 & $\begin{array}{l}\text { Adults } \\
\text { (21-52) }\end{array}$ & $\begin{array}{c}45 \\
\text { (mild asthma) }\end{array}$ & $\mathrm{FEV}_{1} / \mathrm{VC}$ & $\begin{array}{c}71.9 \pm 7.4 \\
\text { to } \\
88.4 \pm 2.7\end{array}$ & +16.5 & +2.96 & \multirow{3}{*}{$\begin{array}{l}8 \text { weeks; standard } \\
\text { deviation } \\
\text { recalculated from } \\
\text { reported standard } \\
\text { error }\end{array}$} & \multirow[t]{3}{*}{ Low } \\
\hline & 2 & $\begin{array}{l}\text { DubiLEY and } \\
\text { SHOGENTSUKOVA, } \\
1973 \text { (2) [18] }\end{array}$ & $\begin{array}{c}\text { USSR } \\
\text { (Russian) }\end{array}$ & 2000 & $\begin{array}{l}\text { Adults } \\
(21-52)\end{array}$ & $\begin{array}{c}70 \\
\text { (moderate } \\
\text { asthma) }\end{array}$ & $\mathrm{FEV}_{1} / \mathrm{VC}$ & $\begin{array}{l}68.7 \pm 10.0 \\
\text { to } \\
80.4 \pm 3.3\end{array}$ & +11.7 & +1.57 & & \\
\hline & 3 & $\begin{array}{l}\text { DUBILEY and } \\
\text { SHOGENTSUKOVA, } \\
1973 \text { (3) [18] }\end{array}$ & $\begin{array}{c}\text { USSR } \\
\text { (Russian) }\end{array}$ & 2000 & $\begin{array}{l}\text { Adults } \\
\text { (21-52) }\end{array}$ & $\begin{array}{c}35 \\
\text { (severe } \\
\text { asthma) }\end{array}$ & $\mathrm{FEV}_{1} / \mathrm{VC}$ & $\begin{array}{l}64.0 \pm 5.3 \\
\quad \text { to } \\
71.4 \pm 7.1\end{array}$ & +7.4 & +1.18 & & \\
\hline 2 & 4 & $\begin{array}{l}\text { KoLESAR et al., } \\
1977 \text { [19] }\end{array}$ & $\begin{array}{l}\text { Czechoslovakia } \\
\text { (Slovak) }\end{array}$ & 1800 & $\begin{array}{l}\text { Adults } \\
\text { (27-39) }\end{array}$ & 15 & $\begin{array}{l}\mathrm{FEV}_{1} \% \\
\text { pred }\end{array}$ & $\begin{array}{c}67.1 \pm 6.6 \\
\text { to } \\
69.9 \pm 7.3\end{array}$ & +2.8 & +0.40 & $\begin{array}{l}2 \text { weeks; data } \\
\text { extracted from a } \\
\text { graphical } \\
\text { presentation }\end{array}$ & Very low \\
\hline 3 & 5 & Boner et al., 1985 [20] & $\begin{array}{c}\text { Italy } \\
\text { (English) }\end{array}$ & 1756 & $\begin{array}{l}\text { Children } \\
\text { (7-14) }\end{array}$ & 14 & $\begin{array}{l}\mathrm{FEV}_{1} \% \\
\text { pred }\end{array}$ & $\begin{array}{l}65.4 \pm 10.8 \\
\text { to } \\
82.5 \pm 17.2\end{array}$ & +17.1 & +1.19 & 8 weeks & Very low \\
\hline \multirow[t]{2}{*}{4} & 6 & $\begin{array}{c}\text { BOBOKHODZHAEV and } \\
\text { SHIRINSKII, } 1990 \text { (1) } \\
{[21]}\end{array}$ & $\begin{array}{c}\text { USSR } \\
\text { (Russian) }\end{array}$ & 1960 & $\begin{array}{l}\text { Adults } \\
(21-56)\end{array}$ & $\begin{array}{c}20 \\
\text { (atopic asthma) }\end{array}$ & $\mathrm{FEV}_{1} / \mathrm{VC}$ & $\begin{array}{c}54.3 \pm 10.3 \\
\text { to } \\
63.2 \pm 17.4\end{array}$ & +8.9 & +0.62 & 4 weeks & \multirow[t]{2}{*}{ Low } \\
\hline & 7 & $\begin{array}{c}\text { BOBOKHODZHAEV and } \\
\text { SHIRINSKII, } 1990 \text { (2) } \\
{[21]}\end{array}$ & $\begin{array}{c}\text { USSR } \\
\text { (Russian) }\end{array}$ & 1960 & $\begin{array}{l}\text { Adults } \\
(21-56)\end{array}$ & $\begin{array}{c}24 \\
\text { (nonatopic } \\
\text { asthma) }\end{array}$ & $\mathrm{FEV}_{1} / \mathrm{VC}$ & $\begin{array}{c}54.1 \pm 10.3 \\
\text { to } \\
75.1 \pm 23.5\end{array}$ & +21 & +1.16 & & \\
\hline \multirow[t]{2}{*}{5} & 8 & $\begin{array}{c}\text { BRIMKULOV, } 1991 \text { (1) } \\
\text { [22] }\end{array}$ & $\begin{array}{c}\text { USSR } \\
\text { (Russian) }\end{array}$ & 3200 & $\begin{array}{l}\text { Adults } \\
\text { (18-58) }\end{array}$ & $\begin{array}{c}38 \\
\text { (mild asthma) }\end{array}$ & $\begin{array}{l}\mathrm{FEV}_{1} \% \\
\text { pred }\end{array}$ & $\begin{array}{l}74.9 \pm 5.5 \\
\text { to } \\
86.4 \pm 7.4\end{array}$ & +11.5 & +1.76 & $\begin{array}{l}4 \text { weeks; standard } \\
\text { deviation } \\
\text { recalculated from }\end{array}$ & \multirow[t]{2}{*}{ High } \\
\hline & 9 & $\begin{array}{c}\text { BRIMKULOV, } 1991 \text { (2) } \\
\text { [22] }\end{array}$ & $\begin{array}{c}\text { USSR } \\
\text { (Russian) }\end{array}$ & 3200 & $\begin{array}{l}\text { Adults } \\
\text { (18-58) }\end{array}$ & $\begin{array}{c}94 \\
\text { (moderate } \\
\text { asthma) }\end{array}$ & $\begin{array}{l}\text { FEV } 1 \% \\
\text { pred }\end{array}$ & $\begin{array}{c}64.5 \pm 17.2 \\
\text { to } \\
73.3 \pm 18.0\end{array}$ & +8.8 & +0.50 & $\begin{array}{l}\text { reported standard } \\
\text { error }\end{array}$ & \\
\hline 6 & 10 & $\begin{array}{l}\text { PIACENTINI et al., } \\
1993 \text { [23] }^{\#}\end{array}$ & $\begin{array}{c}\text { Italy } \\
\text { (English) }\end{array}$ & 1756 & $\begin{array}{l}\text { Children } \\
(10.6 \pm 1.7)\end{array}$ & 20 & $\begin{array}{l}\mathrm{FEV}_{1} \% \\
\text { pred }\end{array}$ & $\begin{array}{c}98.0 \pm 24.0 \\
\text { to } \\
103.0 \pm 34.0\end{array}$ & +5.0 & +0.17 & $\begin{array}{l}2 \text { weeks; data } \\
\text { extracted from a } \\
\text { graphical } \\
\text { presentation }\end{array}$ & Low \\
\hline 7 & 11 & $\begin{array}{l}\text { Simon et al., } \\
1994 \text { [24] }\end{array}$ & $\begin{array}{l}\text { Switzerland } \\
\text { (English) }\end{array}$ & 1560 & $\begin{array}{l}\text { Children } \\
(8-15)\end{array}$ & 14 & $\begin{array}{l}\mathrm{FEV}_{1} \% \\
\text { pred }\end{array}$ & $\begin{array}{c}95.7 \pm 9.7 \\
\text { to } \\
104.0 \pm 14.7\end{array}$ & +8.3 & +0.67 & 5 weeks & Low \\
\hline 8 & 12 & $\begin{array}{l}\text { ALLEGRA et al., } \\
1995 \text { [11] }\end{array}$ & $\begin{array}{l}\text { Italy and Nepal } \\
\text { (English) }\end{array}$ & $\begin{array}{l}4559 \text { and } \\
5050\end{array}$ & $\begin{array}{l}\text { Adults } \\
\text { (23-48) }\end{array}$ & 11 & $\begin{array}{l}\mathrm{FEV}_{1} \% \\
\text { pred }\end{array}$ & $\begin{array}{c}102.7 \pm 20.4 \\
\text { to } \\
104.8 \pm 20.1\end{array}$ & +2.1 & +0.10 & 1 week & Low \\
\hline
\end{tabular}




\begin{tabular}{|c|c|c|c|c|c|c|c|c|c|c|c|c|}
\hline Study & Strata & $\begin{array}{c}\text { Study and year of } \\
\text { publication }\end{array}$ & $\begin{array}{l}\text { Study location } \\
\text { (language of } \\
\text { publication) }\end{array}$ & $\begin{array}{l}\text { Altitude } \\
\text { MASL }\end{array}$ & $\begin{array}{l}\text { Patient } \\
\text { group } \\
\text { adults } \\
\text { versus } \\
\text { children }\end{array}$ & $\begin{array}{l}\text { Subjects } \\
\text { (subgroup) }\end{array}$ & $\begin{array}{l}\text { Outcome } \\
\text { measure } \\
\text { included in } \\
\text { analysis }\end{array}$ & $\begin{array}{l}\text { Observed } \\
\text { change }\end{array}$ & $\begin{array}{c}\text { Mean } \\
\text { difference }\end{array}$ & SMD & $\begin{array}{l}\text { Treatment } \\
\text { duration and } \\
\text { additional } \\
\text { comments }\end{array}$ & $\begin{array}{l}\text { Quality of } \\
\text { evidence }\end{array}$ \\
\hline \multirow[t]{2}{*}{9} & 13 & $\begin{array}{c}\text { BONER et al., } 1995 \text { (1) } \\
\text { [25] }\end{array}$ & $\begin{array}{c}\text { Italy } \\
\text { (English) }\end{array}$ & 1756 & $\begin{array}{l}\text { Children } \\
(6-14)\end{array}$ & $\begin{array}{l}13 \\
\text { (patients on } \\
\text { budesonide) }\end{array}$ & $\begin{array}{l}\text { FEV } 1 \% \\
\text { pred }\end{array}$ & $\begin{array}{c}89.9 \pm 9.9 \\
\text { to } \\
99.5 \pm 10.7\end{array}$ & +9.6 & +0.93 & 6 weeks & Low \\
\hline & 14 & $\begin{array}{l}\text { BONER et al., } 1995 \text { (2) } \\
\text { [25] }\end{array}$ & $\begin{array}{c}\text { Italy } \\
\text { (English) }\end{array}$ & 1756 & $\begin{array}{l}\text { Children } \\
\text { (6-14) }\end{array}$ & $\begin{array}{c}7 \\
\text { (patients on } \\
\text { placebo) }\end{array}$ & $\begin{array}{l}\text { FEV } 1 \% \\
\text { pred }\end{array}$ & $\begin{array}{l}92.9 \pm 6.5 \\
\text { to } \\
95.0 \pm 9.7\end{array}$ & +2.1 & +0.25 & & \\
\hline 10 & 15 & $\begin{array}{c}\text { van Velzen et al., } 1996 \\
\text { [12] }\end{array}$ & $\begin{array}{l}\text { Switzerland } \\
\text { (English) }\end{array}$ & 1560 & $\begin{array}{l}\text { Children } \\
(10-18)\end{array}$ & 16 & $\begin{array}{l}\text { FEV } 1 \% \\
\text { pred }\end{array}$ & $\begin{array}{l}92.1 \pm 23.2 \\
\text { to } \\
97.0 \pm 20.4\end{array}$ & +4.9 & +0.22 & $\begin{array}{l}4 \text { weeks; standard } \\
\text { deviation } \\
\text { recalculated from } \\
\text { reported standard } \\
\text { error }\end{array}$ & Low \\
\hline 11 & 16 & Кокоv, 1996 [26] & $\begin{array}{l}\text { Russian } \\
\text { Federation } \\
\text { (Russian) }\end{array}$ & 1850 & $\begin{array}{l}\text { Children } \\
\text { (9-15) }\end{array}$ & 68 & $\begin{array}{l}\text { FEV } 1 \% \\
\text { pred }\end{array}$ & $\begin{array}{l}74.7 \pm 28.9 \\
\text { to } \\
83.4 \pm 23.1\end{array}$ & +8.7 & +0.33 & $\begin{array}{c}3 \text { weeks; standard } \\
\text { deviation } \\
\text { recalculated from } \\
\text { reported standard } \\
\text { error; study } \\
\text { identified from } \\
\text { www.elibrary.ru }\end{array}$ & High \\
\hline 12 & 17 & $\begin{array}{c}\text { VALLETTA et al., } 1997 \\
{[27]^{\#}}\end{array}$ & $\begin{array}{c}\text { Italy } \\
\text { (English) }\end{array}$ & 1756 & $\begin{array}{l}\text { Children } \\
\text { (9-13) }\end{array}$ & 14 & $\begin{array}{l}\text { FEV } 1 \% \\
\text { pred }\end{array}$ & $\begin{array}{l}82.0 \pm 16.0 \\
\text { to } \\
85.0 \pm 14.0\end{array}$ & +3.0 & +0.20 & $\begin{array}{c}4 \text { weeks; data } \\
\text { extracted from a } \\
\text { graphical } \\
\text { presentation }\end{array}$ & Low \\
\hline 13 & 18 & $\begin{array}{c}\text { GROOTENDORST et al., } \\
2001 \text { [28] }\end{array}$ & $\begin{array}{l}\text { Switzerland } \\
\text { (English) }\end{array}$ & 1560 & $\begin{array}{l}\text { Children } \\
\text { (12-18) }\end{array}$ & 18 & $\begin{array}{l}\text { FEV } 1 \% \\
\text { pred }\end{array}$ & $\begin{array}{c}85.6 \pm 18.9 \\
\text { to } \\
94.8 \pm 17.6\end{array}$ & +9.2 & +0.50 & $\begin{array}{l}10 \text { weeks; } \\
\text { standard deviation } \\
\text { recalculated from } \\
\text { reported standard } \\
\text { error }\end{array}$ & Low \\
\hline 14 & 19 & $\begin{array}{c}\text { Peroni et al., } 2002 \\
\text { [29] }\end{array}$ & $\begin{array}{c}\text { Italy } \\
\text { (English) }\end{array}$ & 1756 & $\begin{array}{l}\text { Children } \\
\text { (7-13) }\end{array}$ & 18 & $\begin{array}{l}\text { FEV } 1 \% \\
\text { pred }\end{array}$ & $\begin{array}{c}100.5 \pm 15.3 \\
\text { to } \\
102.0 \pm 12.3\end{array}$ & +1.5 & +0.11 & $\begin{array}{l}12 \text { weeks; } \\
\text { standard deviation } \\
\text { recalculated from } \\
\text { reported standard } \\
\text { error }\end{array}$ & Low \\
\hline 15 & 20 & $\begin{array}{c}\text { Straub et al., } 2004 \\
{[30]^{\#}}\end{array}$ & $\begin{array}{l}\text { Switzerland } \\
\text { (English) }\end{array}$ & 1560 & $\begin{array}{l}\text { Children } \\
\text { (4-14) }\end{array}$ & 48 & $\begin{array}{l}\text { FEV } 1 \% \\
\text { pred }\end{array}$ & $\begin{array}{c}105.1 \pm 15.9 \\
\text { to } \\
107.0 \pm 18.0\end{array}$ & +1.9 & +0.11 & $\begin{array}{l}4 \text { weeks; mean } \pm \mathrm{SD} \\
\text { recalculated from } \\
\text { the reported } \\
\text { median }(95 \% \mathrm{CI})\end{array}$ & Moderate \\
\hline
\end{tabular}




\begin{tabular}{|c|c|c|c|c|c|c|c|c|c|c|c|c|}
\hline Study & Strata & $\begin{array}{l}\text { Study and year of } \\
\text { publication }\end{array}$ & $\begin{array}{l}\text { Study location } \\
\text { (language of } \\
\text { publication) }\end{array}$ & $\begin{array}{l}\text { Altitude } \\
\text { MASL }\end{array}$ & $\begin{array}{l}\text { Patient } \\
\text { group } \\
\text { adults } \\
\text { versus } \\
\text { children }\end{array}$ & $\begin{array}{l}\text { Subjects } \\
\text { (subgroup) }\end{array}$ & $\begin{array}{l}\text { Outcome } \\
\text { measure } \\
\text { included in } \\
\text { analysis }\end{array}$ & $\begin{array}{l}\text { Observed } \\
\text { change }\end{array}$ & $\begin{array}{c}\text { Mean } \\
\text { difference }\end{array}$ & SMD & $\begin{array}{l}\text { Treatment } \\
\text { duration and } \\
\text { additional } \\
\text { comments }\end{array}$ & $\begin{array}{l}\text { Quality of } \\
\text { evidence }\end{array}$ \\
\hline 16 & 21 & $\begin{array}{l}\text { LOUIE and PARE, } 2004 \\
\text { [31] }\end{array}$ & $\begin{array}{l}\text { Nepal } \\
\text { (English) }\end{array}$ & 4100 & $\begin{array}{l}\text { Adults } \\
(24 \pm 16)\end{array}$ & 5 & PEFR & $\begin{array}{l}558 \pm 43 \\
\text { to } \\
482 \pm 42\end{array}$ & $-76^{\pi}$ & -1.79 & 2 weeks & Low \\
\hline 17 & 22 & $\begin{array}{c}\text { PERONI et al., } 2009 \\
\text { [32] }\end{array}$ & $\begin{array}{l}\text { Italy } \\
\text { (English) }\end{array}$ & 1756 & $\begin{array}{l}\text { Children } \\
(7-14)\end{array}$ & 22 & FEV 1 & $\begin{array}{c}2.75 \pm 0.70 \\
\text { to } \\
2.78 \pm 0.61\end{array}$ & $+0.03^{+}$ & +0.05 & $\begin{array}{l}4 \text { weeks; standard } \\
\text { deviation } \\
\text { recalculated from } \\
\text { reported standard } \\
\text { error }\end{array}$ & Low \\
\hline 18 & 23 & $\begin{array}{c}\text { HUISMANS et al., } 2010 \\
\text { [33] }\end{array}$ & $\begin{array}{c}\text { Nepal } \\
\text { (English) }\end{array}$ & 6410 & $\begin{array}{l}\text { Adults } \\
(39 \pm 9)\end{array}$ & 24 & $\begin{array}{l}\text { FEV } 1 \% \\
\text { pred }\end{array}$ & $\begin{array}{l}104.0 \pm 13.0 \\
\text { to } \\
99.0 \pm 12.0\end{array}$ & -5.0 & -0.40 & 4 weeks & Low \\
\hline \multirow[t]{2}{*}{19} & 24 & $\begin{array}{l}\text { RIJSSENBEEK-NouWENS } \\
\text { et al., } 2012 \text { (1) [34] }\end{array}$ & $\begin{array}{l}\text { Switzerland } \\
\text { (English) }\end{array}$ & 1560 & $\begin{array}{c}\text { Adults } \\
(41.5 \pm 14.5)\end{array}$ & $\begin{array}{c}92 \\
\text { (sensitised to } \\
\text { HDM) }\end{array}$ & $\begin{array}{l}\mathrm{FEV}_{1} \% \\
\text { pred }\end{array}$ & $\begin{array}{l}88.4 \pm 20.4 \\
\text { to } \\
94.2 \pm 20.1\end{array}$ & +5.8 & +0.29 & 12 weeks & High \\
\hline & 25 & $\begin{array}{l}\text { RIJSSENBEEK-NouWENS } \\
\text { et al., } 2012 \text { (2) [34] }\end{array}$ & $\begin{array}{l}\text { Switzerland } \\
\text { (English) }\end{array}$ & 1560 & $\begin{array}{c}\text { Adults } \\
(48 \pm 15.3)\end{array}$ & $\begin{array}{c}45 \\
\text { (nonsensitised } \\
\text { to HDM) }\end{array}$ & $\begin{array}{l}\text { FEV1 } \% \\
\text { pred }\end{array}$ & $\begin{array}{c}86.5 \pm 26.2 \\
\text { to } \\
92.8 \pm 23.1\end{array}$ & +6.3 & +0.26 & & \\
\hline 20 & 26 & SEYs et al., 2013 [35] & $\begin{array}{l}\text { Argentina } \\
\text { (English) }\end{array}$ & 6965 & $\begin{array}{c}\text { Adults } \\
(39.6 \pm 11.3)\end{array}$ & 18 & $\begin{array}{l}\mathrm{FEV}_{1} \% \\
\text { pred }\end{array}$ & $\begin{array}{c}90.2 \pm 12.0 \\
\text { to } \\
98.0 \pm 14.0\end{array}$ & +7.8 & +0.60 & $\begin{array}{l}2 \text { weeks; data } \\
\text { extracted from a } \\
\text { graphical } \\
\text { presentation }\end{array}$ & Low \\
\hline \multirow[t]{2}{*}{21} & 27 & $\begin{array}{l}\text { VERKLEIJ et al., } 2013 \\
\text { (1) [14] }\end{array}$ & $\begin{array}{l}\text { Switzerland } \\
\text { (English) }\end{array}$ & 1560 & $\begin{array}{l}\text { Children } \\
\text { (7-18) }\end{array}$ & $\begin{array}{l}51 \\
\text { (Netherlands } \\
\text { Clinic Davos) }\end{array}$ & $\begin{array}{l}\text { FEV } 1 \% \\
\text { pred }\end{array}$ & $\begin{array}{c}105.8 \pm 3.4 \\
\text { to } \\
106.1 \pm 13.7\end{array}$ & +0.3 & +0.03 & 6 weeks & Moderate \\
\hline & 28 & $\begin{array}{l}\text { VERKLEIJ et al., } 2013 \\
\text { (2) [14] }\end{array}$ & $\begin{array}{l}\text { Switzerland } \\
\text { (English) }\end{array}$ & 1560 & $\begin{array}{l}\text { Children } \\
\text { (7-18) }\end{array}$ & $\begin{array}{c}48 \\
\text { (High Altitude } \\
\text { Clinic Davos) }\end{array}$ & $\begin{array}{l}\mathrm{FEV}_{1} \% \\
\text { pred }\end{array}$ & $\begin{array}{l}99.4 \pm 14.0 \\
\text { to } \\
99.7 \pm 15.5\end{array}$ & +0.3 & +0.02 & & \\
\hline
\end{tabular}

Data are presented as $\mathrm{n}$, range, mean \pm SD or \%, unless otherwise stated. MASL: metres above sea level; SMD: standardised mean difference; FEV1: forced expiratory volume in $1 \mathrm{~s}$; VC: vital capacity; PEFR: peak expiratory flow rate; HDM: house dust mite. ${ }^{\#}$ : study without baseline lung function at sea level, only at first arrival at altitude; ${ }^{\text {I }}$ : L.min ${ }^{-1}$; ${ }^{+}$: L. 
DUBILEY and SHOGENTSUKOVA (1) [18]

DubILEY and SHOgENTSUKova (2) [18

DUBILEY and SHOgENTSUKOVA (3) [18]

KoLESAR et al. [19]

BONER et al. [20]

BoBokHoDZHAEV and SHIRINSKII (1) [21]

BoBOKHODZHAEV and SHIRINSKII (2) [21]

BRIMKULOV (1) [22]

BRIMKULOV (2) [22]

PIACENTINI et al. [23]

SIMON et al. [24]

Allegra et al. [11]

BONER et al. (1) [25]

Boner et al. (2) [25]

KoKov [26]

VAN VELZEN et al. [12]

VALLETTA et al. [27]

GROOTENDORST et al. [28]

Peroni et al. [29]

LOUIE and PARE [31]

StRAUB et al. [30]

Peroni et al. [32]

Huismans et al. [33]

RIJSSENBEEK-NOUWENS et al. (1) [34]

RIJSSENBEEK-NouwENS et al. (2) [34]

SEYs et al. [35]

VERKLEIJ et al. (1) [14]

VERKLEIJ et al. (2) [14]

Overall $\left({ }^{2}=84.7 \%, p=0.000\right)$

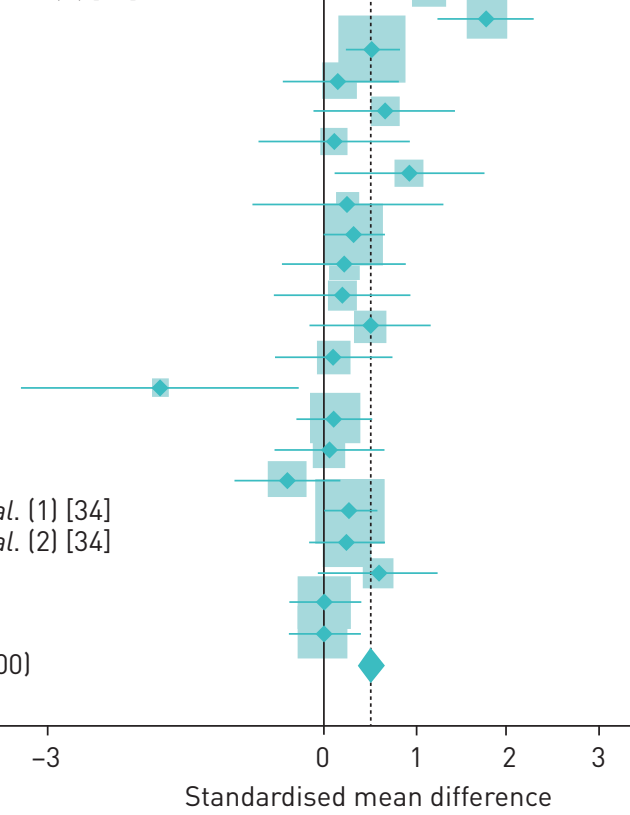

$2.96(2.36-3.56)$

$\begin{array}{ll}.57(1.19-1.95) & 6.41 \\ 1.18(0.67-1.69) & 3.57\end{array}$

$0.40(-0.32-1.13) \quad 1.77$

$1.19(0.38-2.00) \quad 1.41$

$0.62(-0.01-1.26) \quad 2.29$

$1.16(0.54-0.77) \quad 2.46$

$1.76(1.23-2.30) \quad 3.27$

$0.53(0.24-0.82) \quad 10.92$

$0.17(-0.45-0.79) \quad 2.40$

$0.67(-0.10-1.43) \quad 1.59$

$0.10(-0.73-0.94) \quad 1.32$

$0.93(0.12-1.74) \quad 1.40$

$0.25(-0.80-1.31) \quad 0.83$

$0.33(-0.01-0.67) \quad 8.06$

$0.22(-0.47-0.92) \quad 1.91$

$0.20(-0.54-0.94) \quad 1.67$

$0.50(-0.16-1.17) \quad 2.09$

$0.11(-0.55-0.76) \quad 2.16$

$-1.79(-3.31--0.27) \quad 0.40$

$0.11(-0.29-0.51) \quad 5.76$

$0.05(-0.55-0.64) \quad 2.64$

$-0.40(-0.97-0.17) \quad 2.83$

$0.29(-0.00-0.58) \quad 10.95$

$0.26(-0.16-0.67) \quad 5.37$

$0.60(-0.07-1.27) \quad 2.07$

$0.03(-0.36-0.42) \quad 6.13$

$0.02(-0.38-0.42) \quad 5.77$

$0.53(0.43-0.62) \quad 100.00$

\begin{tabular}{lllll}
\hline-3 & 0 & 1 & 2 & 3 \\
& & Standardised mean difference
\end{tabular}

FIGURE 2 Pooled meta-analysis of included studies. Diamonds indicate the standardised mean difference (SMD) and shaded boxes represent the individual study weights.

to 0.59 (95\% CI $0.48-0.69$ ). There was no strong evidence of systematic publication bias (figure 4 ). In addition to the visual pattern displayed in the funnel plot, Begg's test $(p=0.47)$ and Egger's test $(p=0.86)$ showed no clear evidence of publication bias.

\section{Discussion}

This comprehensive quantitative assessment of the effect of HACT on asthma using a standard meta-analytic approach found a statistically significant improvement in lung function following this intervention. Moreover, the positive effect is present among both adults and children with asthma. Particularly noteworthy, in aggregate the magnitude of this effect meets a standard criterion for an MCD, although this benchmark was achieved only among adults. This MCD metric is defined $a \geqslant 0.50$ SMD effect size $[37,38]$. Indeed, this magnitude of

TABLE 2 Re-analysis stratified by altitude, age and duration of high-altitude climatic therapy

\begin{tabular}{|c|c|c|c|c|c|}
\hline & \multirow[t]{2}{*}{ Subjects } & \multirow[t]{2}{*}{ Sets of results } & \multirow[t]{2}{*}{$\operatorname{SMD}(95 \%$ CI $)$} & \multicolumn{2}{|c|}{ Heterogeneity } \\
\hline & & & & $I^{2}$ & p-value ${ }^{\#}$ \\
\hline Overall & 907 & 28 & $0.53(0.43-0.62)$ & 85 & $<0.001$ \\
\hline \multicolumn{6}{|l|}{ Altitude MASL } \\
\hline$\leqslant 2000$ & 717 & 22 & $0.53(0.42-0.64)$ & 85 & $<0.001$ \\
\hline$>2000$ & 190 & 6 & $0.53(0.32-0.74)$ & 88 & 0.02 \\
\hline \multicolumn{6}{|l|}{ Age group } \\
\hline Children & 371 & 14 & $0.24(0.09-0.38)$ & 4 & 0.41 \\
\hline Adults & 536 & 14 & $0.75(0.63-0.88)$ & 90 & $<0.001$ \\
\hline \multicolumn{6}{|c|}{ Treatment duration weeks } \\
\hline$\leqslant 4$ & 437 & 16 & $0.42(0.28-0.55)$ & 74 & $<0.001$ \\
\hline$>4$ & 470 & 12 & $0.63(0.50-0.77)$ & 90 & $<0.001$ \\
\hline
\end{tabular}




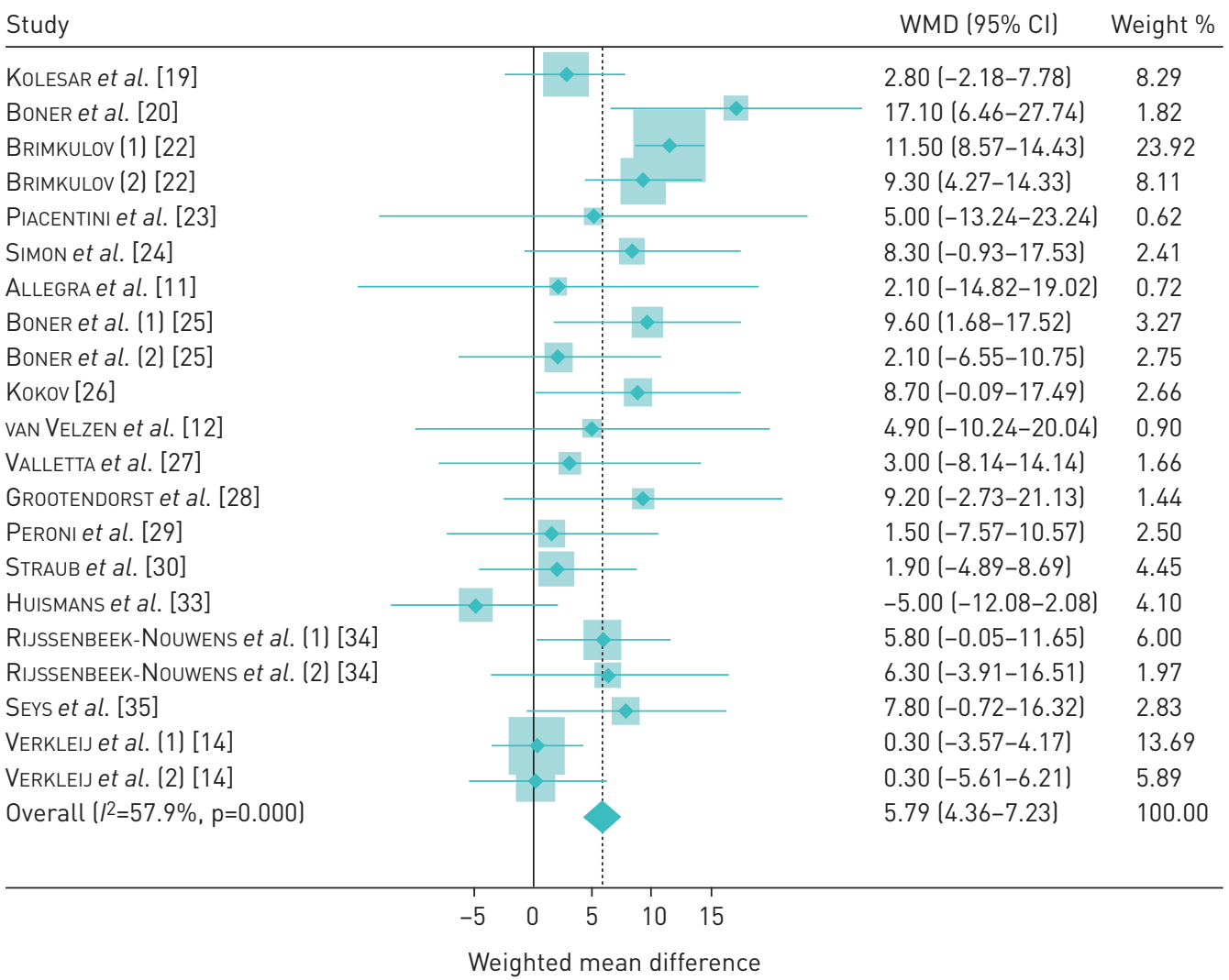

FIGURE 3 Pooled meta-analysis of studies reporting forced expiratory volume in $1 \mathrm{~s} \%$ pred. Diamonds indicate the weighted mean difference (WMD) and the shaded boxes represent the individual weight of each study.

effect is very close to that observed in asthma in clinical trials of moderate doses of inhaled beclomethasone (SMD 0.49, 95\% CI 0.30-0.69) [39]. Using the metric of FEV1 absolute change (standardised to an adult male) available for 21 of the included substudies, HACT appears to be almost as potent compared with the maximum estimated pooled effect of inhaled budesonide for asthma in clinical trials (229 versus $290 \mathrm{~mL}$ ) [40].

Our meta-analysis has a number of strengths. We used a search strategy targeting inclusion of studies published in both English and languages other than English, spanning a wide time range for inclusion. We were conservative in our approach by relying on pulmonary function-defined outcomes as the objective measure of clinical improvement in asthma. By limiting the pool of studies to those $\geqslant 1500$ MASL we also insured that the HACT intervention was at least moderate in its environmental attributes. We acknowledge, however, that some of the studies carried out at the higher altitudes included were not specifically designed to evaluate the effect of HACT, but rather designed as a respite sojourn for disease sufferers. Our retention of study subsets in the analysis rather than collapsing these into single values for each included publication should be considered a study strength rather than a limitation, because this conservative approach enabled estimation of the effect of HACT in different asthma subgroups.

This meta-analysis approach has a number of inherent limitations. We only considered pulmonary function as a measure of clinical effect, although other clinical and functional criteria are used widely to assess asthma. Bronchial hyperreactivity is one of these. Although this was reported in a limited number of the studies that we accessed, there were too few to contribute to an effective meta-analysis strategy. In many but not all of these studies, nonspecific hyperresponsiveness decreased after the intervention, consistent with a salutary effect of HACT on asthma. Another potential limitation in our study is the use of FEV1 as an end-point in children because it may be a poor measure of asthma control, especially in those of younger age [41, 42]. Nevertheless, HACT has been used as ancillary treatment for asthma in the paediatric population, as the number of publications included in this analysis indicate. Furthermore, many of the nonadults included were, in fact, adolescents, thus limiting the likely impact on our findings of using the FEV1 as an asthma measure in nonadults. The studies we included were limited to those conducted at altitude $>1500$ MASL. We have elected to exclude studies $<1500$ MASL to reduce heterogeneity identified in the previous qualitative review [8].

Critically, we did not assess the potential long-term benefit of HACT after return to lower altitude or in combination with other treatments (e.g. standard stepwise pharmacologic therapy). In many of the studies 


\begin{tabular}{|c|c|c|c|c|}
\hline Study and year of publication & Weight & $\operatorname{SMD}(95 \% \mathrm{CI})$ & $I^{2}$ & p-value \\
\hline $\begin{array}{l}\text { DUBILEY and SHOGENTSUKovA, } 1973 \text { [18] } \\
\text { (three groups combined) }\end{array}$ & 12.53 & $0.35(0.25-0.46)$ & 64 & $<0.001$ \\
\hline KoLESAR et al., 1977 [19] & 1.77 & $0.53(0.43-0.63)$ & 85 & $<0.001$ \\
\hline BonER et al., 1985 [20] & 1.41 & $0.52(0.42-0.62)$ & 85 & $<0.001$ \\
\hline $\begin{array}{l}\text { BoвокноDZHAEV and SHIRINSKII, } 1990 \text { [21] } \\
\text { (two groups combined) }\end{array}$ & 4.75 & $0.51(0.41-0.61)$ & 86 & $<0.001$ \\
\hline $\begin{array}{l}\text { BRIMKULOV, } 1991 \text { (2) [22] } \\
\text { (two groups combined) }\end{array}$ & 14.19 & $0.48(0.38-0.59)$ & 84 & $<0.001$ \\
\hline PiACENTINI et al., 1993 [23] & 2.40 & $0.54(0.44-0.64)$ & 85 & $<0.001$ \\
\hline Simon et al., 1994 [24] & 1.59 & $0.53(0.43-0.62)$ & 85 & $<0.001$ \\
\hline AlLegra et al., 1995 [11] & 1.32 & $0.53(0.44-0.63)$ & 85 & $<0.001$ \\
\hline $\begin{array}{l}\text { Boner et al., } 1995 \text { [25] } \\
\text { (two groups combined) }\end{array}$ & 2.23 & $0.53(0.43-0.62)$ & 86 & $<0.001$ \\
\hline van Velzen et al., 1996 [12] & 1.91 & $0.53(0.44-0.63)$ & 85 & $<0.001$ \\
\hline Koкоv, $1996[26]$ & 8.06 & $0.55(0.45-0.65)$ & 85 & $<0.001$ \\
\hline ValLettA et al., 1997 [27] & 1.67 & $0.53(0.44-0.63)$ & 85 & $<0.001$ \\
\hline GROOTENDORST et al., 2001 [28] & 2.09 & $0.53(0.43-0.63)$ & 85 & $<0.001$ \\
\hline PERONI et al., 2002 [29] & 2.16 & $0.54(0.44-0.64)$ & 85 & $<0.001$ \\
\hline Straub et al., 2004 [30] & 5.76 & $0.55(0.46-0.65)$ & 85 & $<0.001$ \\
\hline LoUIE and PARE, 2004 [31] & 0.40 & $0.54(0.44-0.63)$ & 85 & $<0.001$ \\
\hline PERONI et al., 2009 [32] & 2.64 & $0.54(0.44-0.64)$ & 85 & $<0.001$ \\
\hline HuIsMANs et al., 2010 [33] & 2.83 & $0.56(0.46-0.65)$ & 84 & $<0.001$ \\
\hline $\begin{array}{l}\text { RIJSSENBEEK-NouwENS et al., } 2012 \text { [34] } \\
\text { (two groups combined) }\end{array}$ & 16.32 & $0.58(0.47-0.68)$ & 85 & $<0.001$ \\
\hline SEYs et al., 2013 [35] & 2.07 & $0.53(0.43-0.62)$ & 85 & $<0.001$ \\
\hline $\begin{array}{l}\text { VERKLEIJ et al., } 2013 \text { [14] } \\
\text { (two groups combined) }\end{array}$ & 11.90 & $0.60(0.49-0.70)$ & 85 & $<0.001$ \\
\hline
\end{tabular}

included in our analysis the participants were on such therapies. Further, we did not have access to the direct and indirect economic costs of HACT, such that this could be compared to other interventions. We also did not study the effects on asthma of dwelling longer-term at high altitude. Another limitation is that many of the studies that we identified were relatively small in size. This, however, is an argument in favour of a meta-analysis strategy, since statistical power is greater and more subtle associations can be identified when results from multiple small studies can be combined in meta-analysis. Although small studies tend to be impacted by publication bias to a greater degree than larger studies $[15,43]$, we did not find evidence of that in this analysis. Selection bias may be present across all of the selected publications because HACT is often used as a supplementary treatment in relatively stable asthma, whereas patients with severe or difficult to control asthma may be excluded from such investigations. There also may be information bias (disease misclassification) because publications are included that predate current asthma

FIGURE 4 Funnel plot (with pseudo $95 \% \mathrm{Cl}$ of 28 substrata analysed.

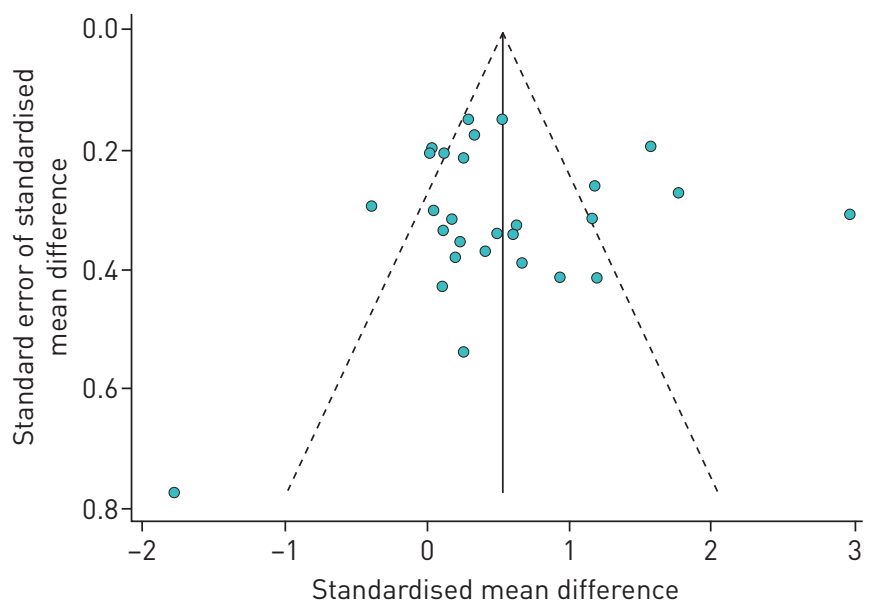


diagnosis and treatment standardisation. Finally, unmeasured confounding cannot be excluded, including unaccounted for climatic or other geographic parameters.

A recent systematic review assessed the effects of HACT on lung function and airway hyperreactivity using a systematic review but without a statistical meta-analytic component [8]. That review concluded that there was insufficient evidence to establish efficacy, invoking heterogeneity of studies and small sample sizes. Of note, that review was limited to studies at altitudes of 1500-2500 MASL published in English and German only, and included longer duration studies that may have added additional heterogeneity. We also found statistically significant heterogeneity in our meta-analysis. Given the variety of different study designs, populations and research methods used, statistical heterogeneity is expected. In contradistinction to this statistical phenomenon, it is important to note that all of the 26 studies carried out at an altitude of $<4000$ MASL consistently observed lung function improvement, a homogeneous direction of effect. The findings of another relatively recent systematic analysis of HACT as an intervention to treat atopic dermatitis, a condition that has some shared attributes with asthma, are also relevant [44]. That review concluded that there was a positive HACT-associated treatment effect. In a broader sense, it also is important to consider whether a meta-analytic approach is appropriate to the data at hand, given that HACT is an environmental intervention that potentially is heterogeneous, and varies by elevation and in its effects among different asthma subgroups. Our results, including the overall findings as illustrated in the forest and funnel plots and in the sensitivity analyses that we performed, indicate that the estimates yielded do provide a useful assessment of the effect of HACT on lung function in asthma.

We found that HACT may be an effective intervention to improve lung function in asthma. Nonetheless, a number of questions remain to be addressed including optimal altitude and duration of treatment, and most importantly, strategies to extend a retained benefit upon return to the home environment and subgroups (e.g. adults rather than children) most likely to benefit from this intervention. Even when geographic conditions allow (i.e. available mountainous areas), HACT is a relatively resource-intensive intervention that may limit its application and be subject to inequalities of access. This clear direction and magnitude of effect shown in this meta-analysis may help to stimulate further inquiry into these outstanding research topics.

\section{Acknowledgements}

Authors would like to thank the authors who have provided additional data on their studies in reply to enquiries (Oktam I. Bobokhodzhaev (Ministry of Health of Tajikistan, Dushanbe, Tajikistan) and Anzor T. Kokov ("Elbrus" Sanatorium, Nalchik, Russia)). We also thank Michaela Hornakova (University Library, Bratislava, Slovakia), Professor Sergey L. Scherbin (Kabardino-Balkarian State University, Nalchik, Russia) and Valery S. Shirinsky (Scientific Institute of Fundamental and Clinical Immunology, Novosibirsk, Russia) for their help in finding original references in the libraries.

\section{References}

1 Global Initiative for Asthma. 2015 Update: Global Strategy for Asthma Management and Prevention. http://ginasthma.org/wp-content/uploads/2016/01/GINA_Report_2015_Aug11-1.pdf Date last accessed: December 10, 2015.

2 Schultze-Werninghaus G. Should asthma management include sojourns at high altitude? Chem Immunol Allergy 2006; 91: 16-29.

3 Vervloet D, Penaud A, Razzouk H, et al. Altitude and house dust mites. J Allergy Clin Immunol 1982; 69: 290-296.

4 Spieksma F, Zuidema P, Leupen M. High altitude and house-dust mites. Br Med J 1971; 1: 82-84.

5 Brimkulov N, Bakirova A, Chaltabaev K. Vliianie vysokogornoi klimatoterapii na funktsiiu kory nadpochechnikov u bolnykh bronkhial'noi astmoi [Effect of high-altitude climate therapy on the adrenal cortex function in patients with bronchial asthma]. Klin Med (Mosk) 1990; 68: 82-85.

6 Meehan R, Duncan U, Neale L, et al. Operation Everest II: alterations in the immune system at high altitudes. J Clin Immunol 1988; 8: 397-406.

7 Moher D, Liberati A, Tetzlaff J, et al. Preferred reporting items for systematic reviews and meta-analyses: the PRISMA statement. Ann Intern Med 2009; 151: 264-269.

8 Massimo T, Blank C, Strasser B, et al. Does climate therapy at moderate altitudes improve pulmonary function in asthma patients? A systematic review. Sleep Breath 2014; 18: 195-206.

9 Benckhuijsen J, van den Bos JW, van Velzen E, et al. Differences in the effect of allergen avoidance on bronchial hyperresponsiveness as measured by methacholine, adenosine $5^{\prime}$-monophosphate, and exercise in asthmatic children. Pediatr Pulmonol 1996; 22: 147-153.

10 Cogo A, Basnyat B, Legnani $\mathrm{D}$, et al. Bronchial asthma and airway hyperresponsiveness at high altitude. Respiration 1997; 64: 444-449.

11 Allegra L, Cogo A, Legnani D, et al. High altitude exposure reduces bronchial responsiveness to hypo-osmolar aerosol in lowland asthmatics. Eur Respir J 1995; 8: 1842-1846.

12 van Velzen E, van den Bos JW, Benckhuijsen JA, et al. Effect of allergen avoidance at high altitude on direct and indirect bronchial hyperresponsiveness and markers of inflammation in children with allergic asthma. Thorax 1996; 51: 582-584.

13 van de Griendt EJ, Verkleij M, Douwes JM, et al. Problematic severe asthma in children treated at high altitude: tapering the dose while improving control. J Asthma 2014; 51: 315-319. 
14 Verkleij M, van de Griendt EJ, Kaptein AA, et al. The prospective association between behavioural problems and asthma outcome in young asthma patients. Acta Paediatr 2013; 102: 504-509.

15 Egger M, Davey Smith G, Schneider M, et al. Bias in meta-analysis detected by a simple, graphical test. BMJ 1997; 315: 629-634.

16 Begg CB, Mazumdar M. Operating characteristics of a rank correlation test for publication bias. Biometrics 1994; 50: 1088-1101.

17 Higgins JPT, Thompson SG, Deeks JJ, et al. Measuring inconsistency in meta-analyses. BMJ 2003; 327: 557-560.

18 Dubilei VV, Shogentsukova EA. Opyt lecheniia bol'nykh bronkhial'noi astmoi $v$ usloviiakh Priel'brus'ia [Experience in treating bronchial asthma under the conditions of the Mount El'brus area]. Vopr Kurortol Fizioter Lech Fiz Kult 1973; 38: 136-140.

19 Kolesar J, Eisner J, Michalicka D, et al. Vplyv roznej nadmorskej vysky na zmenu respiracie u pacientov s astma bronchiale [Influence of different altitudes on respiratory changes in patients with bronchial asthma]. Fysiatr Revmatol Vestn 1977; 55: 268-274.

20 Boner AL, Niero E, Antolini I, et al. Pulmonary function and bronchial hyperreactivity in asthmatic children with house dust mite allergy during prolonged stay in the Italian Alps (Misurina, $1756 \mathrm{~m}$ ). Ann Allergy 1985; 54: $42-45$.

21 Bobokhozhdaev OI, Shirinskii VS. Effektivnost' lecheniia bol'nykh bronkhial'noi astmoi na kurorte Khodzhaobigarm [The efficacy of treating bronchial asthma patients at Khodzhaobigarm health resort]. Vopr Kurortol Fizioter Lech Fiz Kult 1990; 21-26.

22 Brimkulov NN. Vysokogornaia klimatoterapiia bol'nykh bronkhial'noi astmoi [The alpine climatotherapy of bronchial asthma patients]. Ter Arkh 1991; 63: 25-30.

23 Piacentini GL, Martinati L, Fornari A, et al. Antigen avoidance in a mountain environment: influence on basophil releasability in children with allergic asthma. J Allergy Clin Immunol 1993; 92: 644-650.

24 Simon HU, Grotzer M, Nikolaizik WH, et al. High altitude climate therapy reduces peripheral blood T lymphocyte activation, eosinophilia, and bronchial obstruction in children with house-dust mite allergic asthma. Pediatr Pulmonol 1994; 17: 304-311.

25 Boner AL, Comis A, Schiassi M, et al. Bronchial reactivity in asthmatic children at high and low altitude. Effect of budesonide. Am J Respir Crit Care Med 1995; 151: 1194-1200.

26 Kokov AT. [Pulmonary function and surfactant system of the lungs after high-altitude alpine treatment of children with asthma] [Dissertation in Russian]. Stavropol, Stavropol State Medical Academy, 1996.

27 Valletta EA, Piacentini GL, Del Col G, et al. $\mathrm{FEF}_{25-75}$ as a marker of airway obstruction in asthmatic children during reduced mite exposure at high altitude. J Asthma 1997; 34: 127-131.

28 Grootendorst DC, Dahlen SE, Van Den Bos JW, et al. Benefits of high altitude allergen avoidance in atopic adolescents with moderate to severe asthma, over and above treatment with high dose inhaled steroids. Clin Exp Allergy 2001; 31: 400-408.

29 Peroni DG, Piacentini GL, Costella S, et al. Mite avoidance can reduce air trapping and airway inflammation in allergic asthmatic children. Clin Exp Allergy 2002; 32: 850-855.

30 Straub DA, Ehmann R, Hall GL, et al. Correlation of nitrites in breath condensates and lung function in asthmatic children. Pediatr Allergy Immunol 2004; 15: 20-25.

31 Louie D, Pare PD. Physiological changes at altitude in nonasthmatic and asthmatic subjects. Can Respir J 2004; 11 : 197-199.

32 Peroni DG, Bodini A, Loiacono A, et al. Bioimpedance monitoring of airway inflammation in asthmatic allergic children. Allergol Immunopathol 2009; 37: 3-6.

33 Huismans HK, Douma WR, Kerstjens HA, et al. Asthma in patients climbing to high and extreme altitudes in the Tibetan Everest region. J Asthma 2010; 47: 614-619.

34 Rijssenbeek-Nouwens LH, Fieten $\mathrm{KB}$, Bron AO, et al. High-altitude treatment in atopic and nonatopic patients with severe asthma. Eur Respir J 2012; 40: 1374-1380.

35 Seys SF, Daenen M, Dilissen E, et al. Effects of high altitude and cold air exposure on airway inflammation in patients with asthma. Thorax 2013; 68: 906-913.

36 Quanjer PH, Tammeling GJ, Cotes JE, et al. Lung volumes and forced ventilator flows. Report Working Party Standardization of Lung Function Tests, European Community for Steel and Coal. Official Statement of the European Respiratory Society. Eur Respir J Suppl 1993; 16: 5-40.

37 Cohen J. Statistical Power: Analysis for the Behavioral Sciences. 1st Edn. New York, Academic Press, 1977.

38 Copay AG, Subach BR, Glassman SD, et al. Understanding the minimum clinically important difference: a review of concepts and methods. Spine J 2007; 7: 541-546.

39 Adams NP, Bestall JB, Malouf R, et al. Beclomethasone versus placebo for chronic asthma. Cochrane Database Syst Rev 2005; 1: CD002738.

40 Masoli M, Holt S, Weatherall M, et al. Dose-response relationship of inhaled budesonide in adult asthma: a meta-analysis. Eur Respir J 2004; 23: 552-558.

41 Bacharier LB, Strunk RC, Mauger D, et al. Classifying asthma severity in children: mismatch between symptoms, medication use, and lung function. Am J Respir Crit Care Med 2004; 170: 426-432.

42 van Dalen C, Harding E, Parkin J, et al. Suitability of forced expiratory volume in 1 second/forced vital capacity vs percentage of predicted forced expiratory volume in 1 second for the classification of asthma severity in adolescents. Arch Pediatr Adolesc Med 2008; 162: 1169-1174.

43 IntHout J, Ioannidis JP, Borm GF, et al. Small studies are more heterogeneous than large ones: a meta-meta-analysis. J Clin Epidemiol 2015; 68: 860-869.

44 Fieten KB, Weststrate AC, van Zuuren EJ, et al. Alpine climate treatment of atopic dermatitis: a systematic review. Allergy 2015; 70: 12-25. 